\title{
Involvement of mediastinal/intra-abdominal lymph nodes, spleen, liver, and left adrenal in presumed isolated pancreatic tuberculosis: An endoscopic ultrasound study
}

\author{
Surinder S. Rana, Vishal Sharma, Ravi Sharma, Deepak K. Bhasin
}

Department of Gastroenterology, Post Graduate Institute of Medical Education and Research, Chandigarh, India

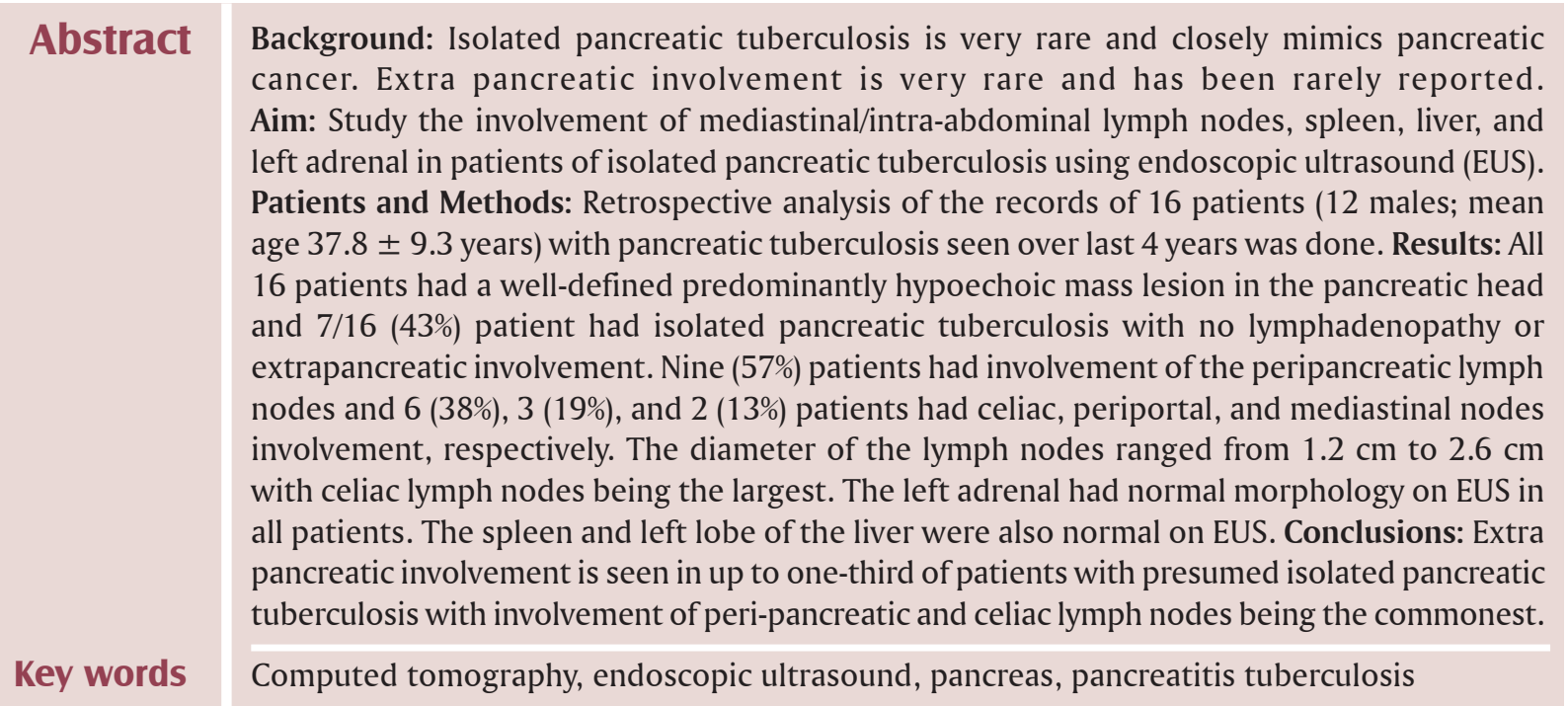

\section{Introduction}

Pancreatic tuberculosis is very rare and usually involves the head and uncinate process of the pancreas. ${ }^{[1-3]}$ The clinical and imaging features of pancreatic tuberculosis closely mimics pancreatic cancer with even vascular invasion being reported with pancreatic tuberculosis. ${ }^{[2,4-7]}$ Most of the cases of isolated pancreatic tuberculosis reported in the literature that had been

\section{Access this article online}

\begin{tabular}{|c|c|}
\hline \multirow{3}{*}{$\begin{array}{l}\text { Website: } \\
\text { www.jdeonline.in }\end{array}$} & Quick Response Code \\
\hline & 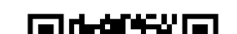 \\
\hline & 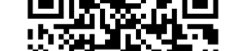 \\
\hline DOI: & \pm 1 \\
\hline $10.4103 / 0976-5042.155236$ & 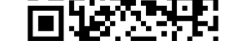 \\
\hline
\end{tabular}

diagnosed on cytology or surgery had localized involvement of pancreas or involvement of peripancreatic lymph nodes only.

Tuberculosis is a systemic disease with the possibility of involvement of multiple organs and lymph nodes. Pancreatic involvement is also seen in the setting of miliary or disseminated tuberculosis and isolated involvement of the pancreas is very rare. ${ }^{[8,9]}$ The involvement of other organs or lymph nodes in pancreatic tuberculosis has not been evaluated by sensitive imaging modalities. Endoscopic ultrasound (EUS) is an excellent imaging modality as it provides high resolution images obtained by a closely placed transducer. It can provide high resolution images of mediastinal, as well as upper intraabdominal lymph nodes along with left adrenal, left lobe of the liver, as well as spleen. Thus, involvement of these organs can be easily ascertained in patients with presumed isolated pancreatic tuberculosis undergoing EUS for evaluation of the pancreatic lesion. The extrapancreatic findings on EUS

Address for correspondence:

Dr. Surinder Singh Rana, Department of Gastroenterology, Postgraduate Institute of Medical Education and Research, Chandigarh - 160 012, India.

E-mail: drsurinderrana@yahoo.co.in 
in presumed isolated pancreatic tuberculosis have not been previously reported. We retrospectively evaluated the EUS data of patients with presumed isolated pancreatic tuberculosis seen at our unit over last 4 years for features of involvement of mediastinal/intraabdominal lymph nodes, spleen, liver, and left adrenal gland.

\section{Patients and Methods}

We retrospectively retrieved and analyzed the collected database of patients diagnosed with pancreatic tuberculosis treated at our unit over last 4 years. The diagnosis of pancreatic tuberculosis was established on the basis of clinical features, radiological findings, cytological findings, and improvement in symptoms with anti-tubercular therapy (ATT). ${ }^{[2]}$ The clinical and investigational features of these patients was retrieved from the recorded database. All patients had undergone contrast enhanced computed tomography (CECT) of the chest and abdomen prior to undergoing EUS. Some of the patients had also undergone positron emission tomography/computed tomography (PET-CT) before undergoing EUS.

After obtaining informed consent, all the patients underwent EUS examination using the linear scanning echoendoscope (EG-3870 UTK linear echoendoscope Pentax Inc, Tokyo, Japan or GF-UCT180; Olympus, Tokyo, Japan). On EUS, the pancreatic lesion was carefully evaluated for the size, location, and appearance along with the presence of any local lymphadenopathy, vascular invasion, and calcifications. The diameter of the common bile duct and pancreatic duct was also noted. EUS-guided fine-needle aspiration (EUS-FNA) was performed from the lesion and material obtained was immediately sent for cytopathological examination. On EUS, presence of mediastinal/intraabdominal lymphadenopathy along with any focal lesion in the spleen, left lobe of the liver or left adrenal gland were also looked for. Any lymph node $>1 \mathrm{~cm}$ in diameter or with suspicious EUS features or being flourodeoxyglucose (FDG) avid was sampled under EUS guidance. Similarly, any suspicious or FDG avid lesion in the liver, spleen, and left adrenal were also sampled and sent for cytopathological examination.

The patients were treated with four drugs ATT (isoniazid $5 \mathrm{mg} / \mathrm{kg} /$ day, rifampicin $10 \mathrm{mg} / \mathrm{kg} /$ day, pyrazinamide $25 \mathrm{mg} / \mathrm{kg} /$ day, and ethambutol $15 \mathrm{mg} / \mathrm{kg} /$ day) and followed-up for disappearance of symptoms. Patients with cholestatic symptoms were also treated with ATT alone and it was decided to place a biliary stent only if there was intractable pruritus, cholangitis, or worsening of cholestatic symptoms after starting ATT.

\section{Results}

Over last 4 years, 16 patients ( 12 males; mean age: $37.8 \pm 9.3$ years) with pancreatic tuberculosis were treated at our unit. On cross-sectional imaging (CECT abdomen and chest) all these patients had isolated pancreatic lesion and on EUS all had a well-defined predominantly hypoechoic mass lesion in the pancreatic head. Seven of these 16 patients (43\%) patient had isolated pancreatic tuberculosis even on EUS with no surrounding or distant lymphadenopathy [Figure 1]. None of the patients had pancreatic calcification. All patients presented with abdominal pain of varying duration associated with loss of appetite. None of these patients had associated fever or night sweats.

Of these 16 patients, 12 (75\%) patients had cholestatic jaundice, but none had cholangitis. The serology for human immunodeficiency virus was negative in all these patients and blood sugars were normal. None of the patients had any clinical or radiological findings of extrapancreatic tuberculosis on CECT. In patients in whom PET scan was done, both the pancreatic as well as extrapancreatic lesions were intensely FDG avid with maximum standardized uptake value ranging from 6 to 22 .

Nine (57\%) patients had enlarged peripancreatic lymph nodes adjacent to the pancreatic head lesion. The celiac, periportal and mediastinal lymph nodes were involved in six (38\%), three (19\%), and two (13\%) patients with five (31\%) patients having involvement of multiple lymph nodes [Figure 2 and 3]. The diameter of the lymph nodes ranged from $1.2 \mathrm{~cm}$ to $2.6 \mathrm{~cm}$ with celiac lymph nodes being the largest. EUS-FNA from the lymph nodes revealed granulomatous inflammation with stain for acid-fast bacilli being negative.

The left adrenal had normal morphology on EUS in all 16 patients and no focal lesion was detected. The spleen and left lobe of the liver also had normal EUS morphology. None of these patients had ascites or pleural effusion. In one patient, a FDG avid lesion was detected in the right lobe of liver and ultrasound guided fine needle aspiration from the hepatic lesion also revealed granulomatous inflammation confirming the diagnosis of tuberculosis. No complications of EUS-FNA were noted in any of these patients.

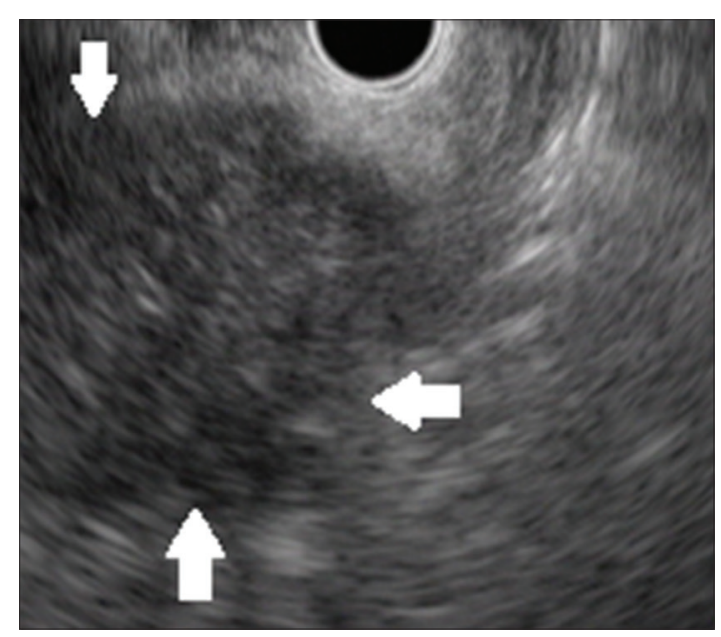

Figure 1: Well-defined hypoechoic lesion in head of the pancreas (arrows) in patient with isolated pancreatic tuberculosis 


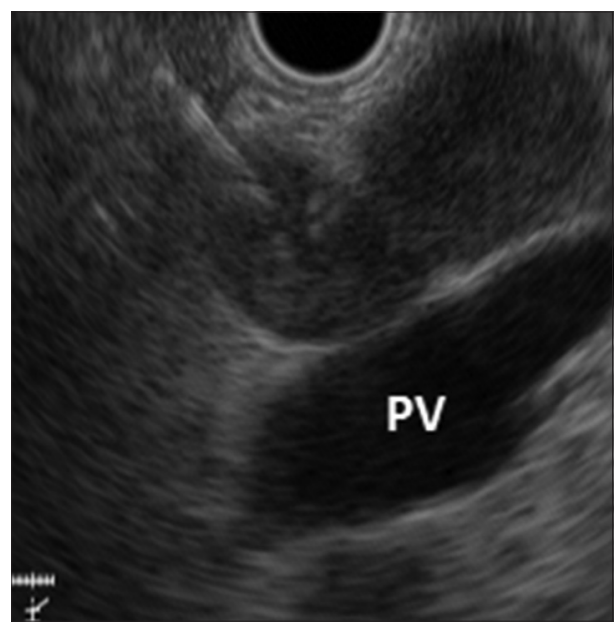

Figure 2: Endoscopic ultrasound-guided fine-needle aspiration from periportal lymph node in patient with pancreatic tuberculosis (PV: Portal vein)

All the patients were treated with standard four-drug ATT and showed a response with disappearance of pain and jaundice within 2 weeks and liver function tests normalized within 2-16 weeks. The bilirubin levels decreased with ATT alone and nobiliary interventions were needed. No adverse effects of ATT were observed in any patient.

\section{Discussion}

Isolated pancreatic tuberculosis is a rare condition, which usually affects the head of the pancreas and is often misdiagnosed as pancreatic malignancy and may result in unwarranted pancreatic resections. ${ }^{[1,2]}$ However, as tuberculosis is a systemic disease, there is a possibility of involvement of multiple organs and local as well as distant lymph nodes. Pancreatic involvement has been described in the setting of miliary or disseminated tuberculosis ${ }^{[8,9]}$ The involvement of other organs or lymph nodes in presumed isolated pancreatic tuberculosis has not been evaluated by sensitive imaging modalities. In the current study, we looked at the involvement of mediastinal/intraabdominal lymph nodes, spleen, liver, and left adrenal gland on EUS in patients with presumed isolated pancreatic tuberculosis.

We found that extrapancreatic involvement by tuberculosis is seen in up to one-third of patients with presumed isolated pancreatic tuberculosis and involvement of peripancreatic and celiac lymph nodes is the commonest. Involvement of other intraabdominal solid parenchymal organs was not commonly seen with only one patient having involvement of liver and none of the patients having splenic or left adrenal involvement. None of the patients had ascites or pleural effusion also. A study of 32 patients with pancreatic tuberculosis found involvement of peripancreatic lymph nodes in 29 (90.6\%) patients on CT abdomen. ${ }^{[10]}$ In this study, extrapancreatic involvement was not reported. A study from China involving 13 patients with pancreatic tuberculosis reported

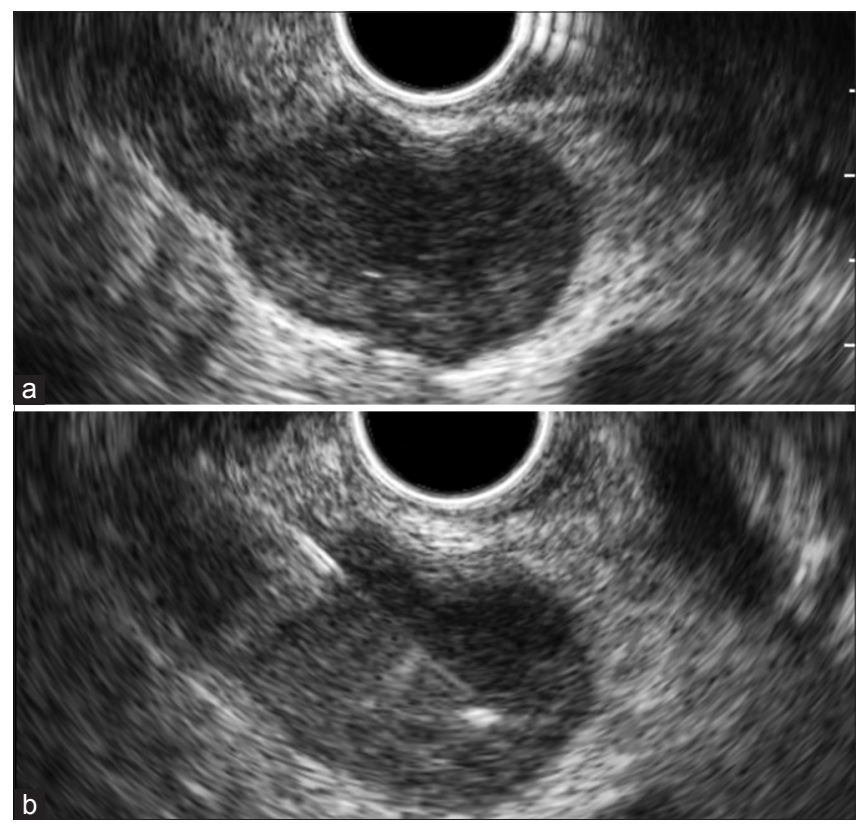

Figure 3: (a) Subcarinal lymph node in patient with pancreatic tuberculosis. (b) Endoscopic ultrasound-guided fine-needle aspiration from subcarinal lymph node

extrapancreatic involvement in three $(23 \%)$ patients. Other studies have also reported rare occurrence of extrapancreatic involvement by pancreatic tuberculosis with many patients undergoing resection for presumed pancreatic cancer. ${ }^{[7,11]}$

In conclusion, using EUS, we found that extrapancreatic involvement by tuberculosis is seen in up to one-third of patients with presumed isolated pancreatic tuberculosis and involvement of peripancreatic and celiac lymph nodes being the most common. Involvement of other intraabdominal solid parenchymal organs is not commonly seen with pancreatic tuberculosis.

\section{References}

1. Rana SS, Bhasin DK, Srinivasan R, Sampath S, Mittal BR, Singh K. Distinctive endoscopic ultrasound features of isolated pancreatic tuberculosis and requirements for biliary stenting. Clin Gastroenterol Hepatol 2012;10:323-5.

2. Rana SS, Sharma V, Sampath S, Sharma R, Mittal BR, Bhasin DK. Vascular invasion does not discriminate between pancreatic tuberculosis and pancreatic malignancy: A case series. Ann Gastroenterol 2014;27:395-8.

3. Sharma V, Chhabra P, Rana SS, Bhasin DK. Pancreatic tuberculosis: Look at the kidney! Dig Liver Dis 2015;47:e1.

4. Rana SS, Chaudhary V, Gupta N, Sampath S, Mittal BR, Bhasin DK. Pancreatic tuberculosis presenting as an unusual head mass. Endoscopy 2013;45 Suppl 2 UCTN:E317-8.

5. Rana SS, Bhasin DK, Gupta N, Singh K. Pancreatic tuberculosis with common bile duct and pancreatic duct dilatation. Endoscopy 2011;43 Suppl 2 UCTN:E282-3.

6. Rana SS, Bhasin DK, Rao C, Singh K. Isolated pancreatic tuberculosis mimicking focal pancreatitis and causing segmental portal hypertension. JOP 2010;11:393-5.

7. Weiss ES, Klein WM, Yeo CJ. Peripancreatic tuberculosis mimicking pancreatic neoplasia. J Gastrointest Surg 2005;9:254-62. 
8. Sharma SK, Mohan A. Extrapulmonary tuberculosis. Indian J Med Res 2004;120:316-53.

9. Haddad F. Abdominal tuberculosis. Dis Colon Rectum 1988;31:333.

10. Nagar AM, Raut AA, Morani AC, Sanghvi DA, Desai CS, Thapar VB. Pancreatic tuberculosis: A clinical and imaging review of 32 cases. J Comput Assist Tomogr 2009;33:136-41.

11. Ozden I, Emre A, Demir K, Balci C, Poyanli A, Ilhan R. Solitary pancreatic tuberculosis mimicking advanced pancreatic carcinoma. J Hepatobiliary
Pancreat Surg 2001;8:279-83.

How to cite this article: Rana SS, Sharma V, Sharma R, Bhasin DK Involvement of mediastinal/intra-abdominal lymph nodes, spleen, liver, and left adrenal in presumed isolated pancreatic tuberculosis: An endoscopic ultrasound study. J Dig Endosc 2015;6:15-8.

Source of Support: Nil, Conflict of Interest: None declared.

\section{"Quick Response Code" link for full text articles}

The journal issue has a unique new feature for reaching to the journal's website without typing a single letter. Each article on its first page has a "Quick Response Code". Using any mobile or other hand-held device with camera and GPRS/other internet source, one can reach to the full text of that particular article on the journal's website. Start a QR-code reading software (see list of free applications from http://tinyurl.com/ yzlh2tc) and point the camera to the QR-code printed in the journal. It will automatically take you to the HTML full text of that article. One can also use a desktop or laptop with web camera for similar functionality. See http://tinyurl.com/2bw7fn3 or http://tinyurl.com/3ysr3me for the free applications. 